\title{
Sensitive high resolution radio imaging of supernova remnants in the M82 starburst
}

\author{
D. Fenech*, A. Pedlar, R. Beswick, T. W. B. Muxlow, M. K. Argo \\ Jodrell Bank Observatory \\ E-mail: Danielle.Fenech@postgrad.manchester.ac.uk
}

\begin{abstract}
High resolution radio observations of the nearby starburst galaxy $\mathrm{M} 82$ at both $1.6 \mathrm{GHz}$ and $5 \mathrm{GHz}$ are presented. The combination of simultaneous Global VLBI and MERLIN observations of M82, made on the 3rd Mar. 2005, have produced the first detailed observations of the majority of the supernova remnants within the central starburst of M82 at a frequency of $1.6 \mathrm{GHz}$. These data also provide the fifth epoch of milliarcsec resolution images of the four most compact sources, allowing monitoring of their evolution over a 19 year timeline. In addition, MERLIN $5 \mathrm{GHz}$ observations over an 8 day period have been used to study the expansion of ten of the supernova remnants within the central starburst.
\end{abstract}

From planets to dark energy: the modern radio universe

October 1-5 2007

University of Manchester, Manchester, UK

*Speaker. 


\section{Introduction}

M82 is considered an archetypal starburst galaxy, and as one of the closest (distance $\sim 3.2 \mathrm{Mpc}$ ), provides an ideal opportunity to study a star forming environment in detail. The first detailed radio studies of M82 [1] revealed a number of compact sources in the central region first identified as either radio supernovae or supernova remnants (SNR) [2,3]. Their subsequent lack of variability confirmed their identity as SNR. Further MERLIN (Multi-Element Radio-Linked Interferometer Network) and VLA (Very Large Array) observations have shown the central kiloparsec of M82 to contain at least 50 compact sources including SNR and HII regions all of which are resolved by either MERLIN [4, 5, 6] and/or VLBI [7, 8, 9].

\section{1.6 GHz global VLBI \& MERLIN observations}

Sixteen radio stations across America and Europe were used to make global VLBI observations of M82 at $1.6 \mathrm{GHz}$ on the 3rd Mar. 2005. These provide a fifth epoch of observations at this frequency and have been used along side EVN (1986 and 1997), and global VLBI (1998 and 2001) observations covering a nineteen year timeline, to study the expansion and evolution of the four most compact supernova remnants within M82 at high angular resolutions. The shell-like source 43.31+59.6 (see Fig. 1), has been studied using the previous epochs, yielding an expansion velocity of $8000-10000 \mathrm{~km} \mathrm{~s}^{-1}[7,8,9]$. Comparison of the most recent observations with data from 2002 , provides a velocity of $\sim 7900 \mathrm{~km} \mathrm{~s}^{-1}$, slightly lower than previously observed and perhaps indicative of deceleration. Using the measured sizes from integrated annular profiles at each epoch, a lower limit of 0.55 has been calculated for the deceleration parameter.

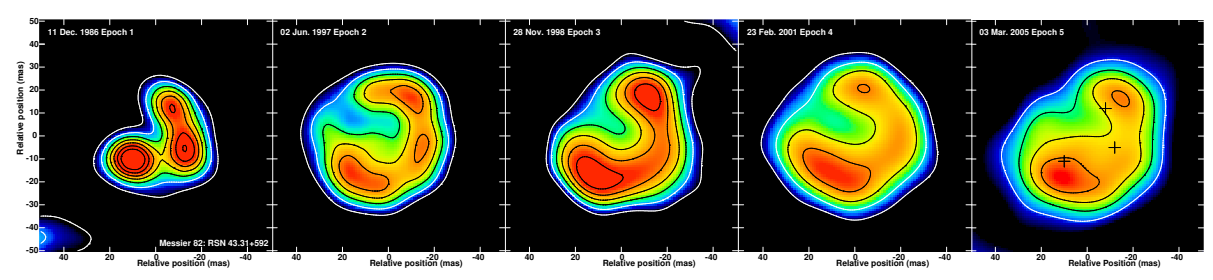

Figure 1: Contour and colour-scale images of the SNR 43.31+59.2 from all five epochs of $1.4 \mathrm{GHz}$ (first epoch only) and $1.6 \mathrm{GHz}$ observations. Each image is restored with a 15 mas beam. Contours are plotted at $-1,1,2, . .10 \times 0.35 \mathrm{mJy}_{\text {beam }}{ }^{-1}$. The colour-scale is linear ranging from 0.1 to $2.2 \mathrm{mJybeam}^{-1}$ for the first three epochs and from 0.1 to 1.7 mJybeam $^{-1}$ for the latter two.

The most compact source within M82, 41.95+57.5, shows behaviour less typical of a SNR. It has a distinct bi-polar structure, unlike for example the radio shell of $43.31+59.6$. The EVN observations did not completely resolve this source, hence, earlier global VLBI observations at the same frequency have been used to study the evolution of this source, which have shown it to be expanding at $\sim 1500-2500 \mathrm{~km} \mathrm{~s}^{-1}[10,7,8,9]$. However, determination of any expansion using the most recent epoch of observations has been hindered by the significant small-scale evolution of both the flux density and morphology of this source. 


\section{Global VLBI and MERLIN data combination}

The MERLIN array was used to observe M82 at $1.6 \mathrm{GHz}$, simultaneous to the global VLBI observations, enabling the combination of the two data sets to produce high fidelity images of the central kiloparsec of M82 for the first time at this frequency (an example of which is shown in Fig. 2). The data have been fully imaged with angular resolutions ranging from 3-130 mas, revealing the detailed structure of 37 of the SNR and HII regions within M82, including two new sources.

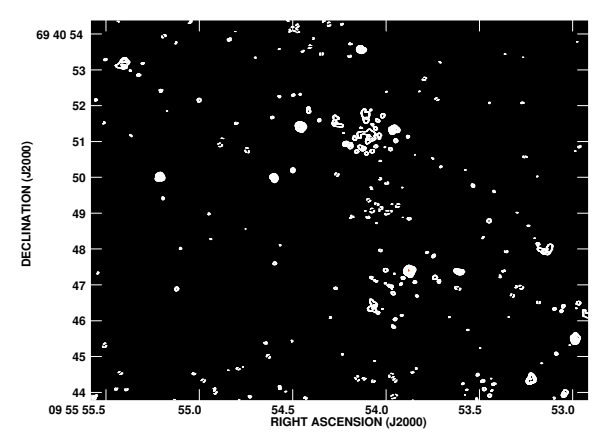

Figure 2: An example field of the combined 1.6 GHz observations, convolved with a 35 mas beam. Contours are plotted at $-1,1,2,3 \ldots 19,20 \times 0.196 \mathrm{mJy}_{\text {beam }}^{-1}(3 \sigma \mathrm{rms})$ and the grey-scale is linear, ranging from 0.07 to $50 \mathrm{mJy} \mathrm{beam}^{-1}$.

\section{5 GHz Deep MERLIN Observations}

Observations of M82 at a frequency of $4.994 \mathrm{GHz}$ between the 1 and 28 Apr. 2002 were made using the MERLIN array. This deep integration observation provided over 175 hours on source and resulted in an image rms sensitivity of $\sim 17 \mu \mathrm{Jy}_{\text {beam }}{ }^{-1}$. These data have been used to produce detailed images of 55 sources in the central region of M82 revealing shell and partial shell structures. The measured diameters of the 37 detected SNR range from 0.4 to $7.8 \mathrm{pc}$ with a mean size of $3.0 \mathrm{pc}$ (See [6]).

Previous MERLIN observations made in Jul. 1992 (see [4]) over a two day period (total 36 hours) have been used to produce astrometrically aligned images to investigate the expansion and variability of the observed SNR. This has provided expansion velocities for ten of the SNR over a ten year baseline. The velocities range from $1500-10500 \mathrm{~km} \mathrm{~s}^{-1}$, confirming both the low expansion of 41.95+57.5 and that of the shell-like SNR 43.31+59.6 observed at Global VLBI resolutions (see $[7,8,9]$ ). The highest measured velocity is of the SNR 43.18+58.2, which also shows a distinct almost complete shell-structure.

Observations of the SNR population at both $1.6 \mathrm{GHz}$ and $5 \mathrm{GHz}$ have been used to determine the supernova rate using a number of methods. These provide measurements of $v_{S N} \sim 0.07 / \mathrm{yr}$, which can be used to infer star formation rates of $\sim 1.8 \mathrm{M}_{\odot} / \mathrm{yr}$. 


\section{Conclusions}

Global VLBI observations of M82 have provided a fifth epoch of observations to study the detailed evolution of the compact sources over a nineteen year timeline. These have provided expansion velocities for the SNR 43.31+59.6 and suggest that this source may be beginning to decelerate. The combination of these data with simultaneous MERLIN observations has revealed the structure of 37 of the discrete sources using resolutions of $\sim 3-130$ mas. Comparison of extremely sensitive $5 \mathrm{GHz}$ MERLIN observations with previous data has enabled the measurement of expansion velocities for ten of the SNR within M82 providing values ranging from 1500-10500 $\mathrm{km} \mathrm{s}^{-1}$.

\section{Acknowledgments}

MERLIN is a national facility run by the University of Manchester on behalf of PPARC. The EVN is a joint facility of European, Chinese, South African and other radio astronomy institutes. The VLBA is operated by the National Radio Astronomy Observatory, a facility of the National Science Foundation, operated under cooperative agreement by Associated Universities, Inc. DF acknowledges financial support via a PPARC funded studentship.

\section{References}

[1] P. P. Kronberg, P. N. Wilkinson, High-resolution, multi-frequency radio observations of M82, ApJ (1975) 300430

[2] S.W. Unger, A. Pedlar, D.J. Axon, P.N.Wilkinson, P.N. Appleton, Young supernovae in the starbust galaxy M82, MNRAS (1984) 211783

[3] P. P. Kronberg, P. Biermann, F. R. Schwab, The nucleus of M82 at radio and X-ray bands - Discovery of a new radio population of supernova candidates, ApJ (1985) 291693

[4] T.W.B. Muxlow, A. Pedlar, P.N. Wilkinson, D.J. Axon, E.M. Sanders, A.G. de Bruyn, The Structure of Young Supernova Remnants in M82, MNRAS (1994) 266455

[5] T.W.B. Muxlow, A. Pedlar, A. Riley, J. McDonald, R.J. Beswick, K.A. Wills, Deep radio imaging with MERLIN of the supernova remnants in M82, in proceedings of IAU Colloquim 192, Springer Proceedings in Physics (2005) 192227

[6] D.Fenech, A. Pedlar, T.W.B. Muxlow, R.J. Beswick, M.K. Argo, K.A. Wills, in prep.

[7] A. Pedlar, T.W.B. Muxlow, M.A. Garret, P.J. Diamond, K.A. Wills, P.N. Wilkinson, W. Alef, VLBI observations of supernova remnants in Messier 82, MNRAS (1999) 307761

[8] A.R. McDonald, T.W.B. Muxlow, A. Pedlar, M.A. Garret, K.A. Wills, S.T. Garrington, P.J. Diamond, P. N. Wilkinson, Global Very Long Baseline Interferometry observations of compact radio sources in M82, MNRAS (2001) 322100

[9] R.J. Beswick, J.D. Riley, I. Marti-Vidal, A. Pedlar, T.W.B. Muxlow, A.R. McDonald, K.A. Wills, D. Fenech, M.K. Argo, 15 years of Very Long Baseline Interferometry observations of two compact radio sources in Messier 82, MNRAS (2006) 3691221

[10] W.M. Trotman, VLBI Observations of 41.9+58: An intermediate-age radio supernova in the starburst nucleus of M82, MSc Thesis, University of Manchester (1996) 